\title{
Randomised controlled trial of a brief intervention targeting predominantly non-verbal communication in general practice consultations
}

\begin{abstract}
\section{Background}

The impact of changing non-verbal consultation behaviours is unknown.

Aim

To assess brief physician training on improving predominantly non-verbal communication.

\section{Design and setting}

Cluster randomised parallel group trial among adults aged $\geq 16$ years attending general practices close to the study coordinating centres in Southampton.

\section{Method}

Sixteen GPs were randomised to no training.

or training consisting of a brief presentation

of behaviours identified from a prior study lacronym KEPe Warm: demonstrating

Knowledge of the patient; Encouraging [backchannelling by saying 'hmm', for example]; Physically engaging [touch, gestures, slight lean]; Warm-up: cool/professional initially, warming up, avoiding distancing or non-verbal cut-offs at the end of the consultation); and encouragement to reflect on videos of their consultation. Outcomes were the Medical Interview Satisfaction Scale (MISS) mean item score (1-7) and patients' perceptions of other domains of communication.

\section{Results}

Intervention participants scored higher MISS overall $10.23,95 \%$ confidence interval $[\mathrm{Cl}]=0.06$ to 0.41 ), with the largest changes in the distress-relief and perceived relationship communication is highlighted as the core of good practice, yet the evidence to inform training needs of health professionals of what they need to do in consultations to maximise effective verbal and non-verbal communication is limited. Beck et al systematically reviewed the verbal and nonverbal behaviours likely to be associated with outcomes. ${ }^{1}$ Verbal behaviours included empathy, reassurance, support, patient-centred questioning, history taking, explanations, humour, psychosocial talk, time in health education and information sharing, friendliness, courtesy, and summarisation and clarification. ${ }^{1}$ Nonverbal behaviours included head nodding. forward lean, direct body orientation, uncrossed legs and arms, arm symmetry, and gaze. An observational study identified important domains of patients' perceptions of communication: a communication and partnership approach, interest in the patient's life, health promotion, a positive approach, and a personal relationship; each of which strongly predicts different outcomes. ${ }^{2,3}$ However, it is very difficult to know what features of verbal and nonverbal communication are most important in modifying patient perceptions because
\end{abstract} subscales. Significant improvement occurred in perceived communication/partnership 10.29 , $95 \% \mathrm{Cl}=0.09$ to 0.49 ) and health promotion (0.26, $95 \% \mathrm{Cl}=0.05$ to 0.46 ). Non-significant improvements occurred in perceptions of a personal relationship, a positive approach, and understanding the effects of the illness on life.

\section{Conclusion}

Brief training of GPs in predominantly nonverbal communication in the consultation and reflection on consultation videotapes improves patients' perceptions of satisfaction, distress, a partnership approach, and health promotion.

\section{Keywords}

communication; consultation; general practice nonverbal communication; patient satisfaction.

\section{INTRODUCTION}

The consultation is central to all medical encounters and patient-centred

P Little, BA, MSc, FMedSci, FRCGP, MRCP, MD, professor of primary care research; J Kelly, BSc, MSc, study manager; $\mathbf{H}$ Everitt, BSc, MSc, PhD, MRCGP, associate professor, Primary Medical Care Group, Community Clinical Sciences Division, Faculty of Medicine, University of Southampton,

Southampton. P White, MSc, MBBS, GP. Nightingale Surgery, Romsey. S Mercer, BSc, MSc, PhD, FRCGP, professor of primary care research, General Practice \& Primary Care, Institute of Health and Wellbeing, University of Glasgow, Glasgow.

\section{Address for correspondence}

Paul Little, Primary Care Group, Primary Care these studies vary greatly in the choice and measurement of exposure and outcome variables

A meta-analysis in 2004 of 106 observational studies and 21 experimental interventions concluded that interventions to improve verbal communication were likely to improve satisfaction but there was very little clarity about what components of intervention were important, little evidence for interventions to modify non-verbal communication, and no clear evidence of an effect on health outcomes. ${ }^{4}$ Very brief patient activation interventions have variable outcomes ${ }^{5}$ but on average no major impact. As part of their training, GPs are used to seeing and learning from videos, but there are few trial data documenting the effect of this. A Cochrane Review of trials to modify patient-centredness updated in 2012 concluded that studies using complex interventions have mixed effects on behaviour and patient satisfaction (risk ratio $=0.99$ for satisfaction as a dichotomous outcome and standard deviation [SD] $=0.35$ for continuous outcomes), with small positive effects on health status. ${ }^{6}$ However, for the best proxy of perceived communication (satisfaction), none of the studies reported addressing non-verbal skills, and nearly all involved several hours of training with expert facilitators (even 'brief' training was

and Population Sciences Unit, Faculty of Medicine, University of Southampton, Aldermoor Health Centre, Southampton S016 5ST, UK.

E-mail: p.littleassoton.ac.uk

Submitted: 23 August 2014; Editor's response: 12 October 2014; final acceptance: 21 October 2014

\section{CBritish Journal of General Practice}

This is the full-length article (published online 26 May 2015) or an abridged version published in print. Cite this article as: Br J Gen Pract 2015; DOI: 10.3399/bjgp15X685237 


\section{How this fits in}

Previous observational studies and experimental interventions suggest that those used to improve GPs' verbal communication are likely to improve patients' satisfaction, but many interventions are intensive, and there is little evidence for those aimed at modifying non-verbal communication. This study demonstrates that very brief training of GPs in predominantly non-verbal elements of communication in the consultation combined with encouragement to reflect on consultation videotapes, is likely to improve patients' perception of satisfaction distress, a partnership approach, and health promotion.

categorised as up to 10 hours of external facilitation). ${ }^{6}$

The authors have previously explored the aspects of verbal and non-verbal communication that are likely to be important in a study of 275 consultations with 25 GPs (see the accompanying article in this Journall. ${ }^{7}$ The results suggested that non-verbal behaviour (such as appropriate gestures and physical contact) and nonspecific verbal behaviour (such as backchannelling and social talk) are important, and that particular care needs to be taken towards the end of the consultation to avoid non-verbal cut-offs and distancing. This study reports the impact on patients perceptions of communication associated with providing a summary of these findings and GPs reviewing and reflecting on their videotaped consultations.

\section{METHOD}

At the time of developing the initial questionnaires to measure patientcentredness, ${ }^{2,3}$ an associated randomised trial was approved by the ethics committee, and prior to the requirements to register trials. The trial was originally designed as an individually randomised trial where patients would be randomised to a more empathic or less empathic encounter, and a more positive or less positive approach.3,8 Two issues forced a change in design: the main issue was that once the trial commenced (in 2001) of the first four GPs who tried randomising their behaviour, only one of the GPs felt able to do so and recruitment was very slow. Therefore the original design was unfeasible and was eventually abandoned. A second issue was that it became apparent from the observational data that it was not possible to fully understand the key modifiable variables that could improve perception of communication..$^{1,9}$ The ethics committee gave approval in 2006 for both further data collection and analysis of the observational data and modification of the design to a cluster randomised trial, reported here, to be based on the findings of the observational phase. The length of time between approval of the changes to the protocol and completion of both the observational study and then recommencing the trial, reflect both the lack of substantive funding of the project (hence part-time data collection), and the time-consuming nature of the observational data analysis of videotapes required before the trial could recommence.

Given the difficulties experienced in randomising individual consultations the modified trial design was a cluster randomised trial: GPs still available who had agreed to the initial observational phase of the study were randomised to receive the brief training intervention or no training intervention. GPs then recruited patients and patients reported their perceptions of communication in the consultation in a post-consultation questionnaire.

GPs were all initially contacted by the local postgraduate tutor in Southampton and the immediate area. Twenty-five GPs close to the study centre who took part in the observational phase of this study had originally agreed to take part in the second phase but there was considerable delay in processing the observational data (which was very intensive and dependent on the availability of staff). Hence 16 of the original GPs were available for randomisation. Of the nine who were not available, three had retired, two had moved away, one was just back from maternity leave, and the others did not reply. One of the research team made an anonymised list of GPs and a second member of the research team made allocations blind to the name of the GP into two groups - intervention and control - using computer-generated random numbers. The control group was also offered the intervention but delayed until after the end of this trial, and were told that there would be training in brief communication skills but not the content of the training.

Participants were any adult patient attending their GP who had agreed to participate in the study and were able and willing to consent to study procedures. Excluded were those who were unable to consent or complete questionnaires (for example, because of severe mental illness, 


\section{Box 1. KEPe Warm}

1. Knowing: the patient's history, social talk

. Encouraging: back-channelling ('hmm', 'ahh')

3. Physically engaging: hand gestures, appropriate contact, slight lean towards the patient

4. Warm: Warm up

- Cooler and professional but supportive at the beginning of the consultation

- Avoid coolness, dominance, patronising. non-verbal cut-offs lfor example, looking away from the patient) at the end of the consultation

severe distress, very unwell generally, and difficulty reading or writing).

The aim was to provide simple feedback from the prior observational study and to provide a memorable acronym to focus thinking about key aspects of the consultation when GPs reviewed their consultations. The acronym 'KEPe Warm' was used (Box 1).

A brief (5-10 minute) presentation of the

Figure 1. CONSORT flow diagram.

MISS = Medical Interview Satisfaction Scale. results from the first phase was made by a medical student to each intervention GP using a standardised handout of the slides, and a 'KEPe Warm' summary sheet. Videotaping took 1-2 minutes for each GP to set up for each surgery both in the previous observational study and the trial. Usually two or three surgeries were needed to get the 15 consultations. The GPs were given their videotape from the observational study and were encouraged to watch as many of the consultations as they felt useful in their own time; but how many they decided to watch was left completely up to them. GPs reported that key limitations in consultations were apparent very quickly: in fewer than five consultations. GPs were asked to pick out three things that they would want to change about their behaviour lwhich could include but did not have to include the KEPe Warm items). They had 1-3 weeks (dependent on the availability of research staff, availability of surgeries, and with sufficient time to reflect on and to have started implementing their changes) before the intervention group were re-videoed in up to 15 consultations each when data collection commenced. The control group were not shown any presentation nor given any summary sheets.

\section{Data collection}

Patients completed a post-consultation questionnaire that was designed to be completed immediately after the consultation, although it could be taken home and returned via freepost.

Patients completed the validated Medical Interview Satisfaction Scale (MISS) to explore the patient's satisfaction with the GP's approach during their consultation as well as their perception of communication (the primary outcome)..$^{10}$ MISS was not only well validated but likely to be relevant based on the prior observational study for the KEPe elements of KEPe Warm. ${ }^{7}$ Patients' perceptions of communication scales were developed based on Stewart's five principles of patient-centredness and validated in the previous study, ${ }^{2,3}$ documenting five domains of patient perception lcommunication, partnership, interest in life, health promotion, and a positive approach).

Patients' perception of enablement was documented using the previously developed instrument. ${ }^{11}$

Other potential confounding factors were also documented, such as the nature of the problem, medication, and other medical problems as well as sociodemographic details of the patient including age, sex, occupation, marital status, and years in 


\section{Table 1. Characteristics of study patients}

\begin{tabular}{lcc} 
Characteristics & Control & Intervention \\
\hline Mean age, years (SD) & $56(21)$ & $51(23)$ \\
Paid work, $n(\%)$ & $39 / 92(42)$ & $39 / 97(40)$ \\
Married, $n(\%)$ & $61 / 89(69)$ & $61 / 97(63)$ \\
Mean years in full time education since age 10 (SD) & $9(6)$ & $9(6)$ \\
Mean number of times visited GP in last year (SD) & $4.5(3.6)$ & $4.8(4.9)$ \\
On medication prior to consultation, $n(\%)$ & $66 / 91(73)$ & $65 / 97(67)$ \\
HADS depression score (SD) & $5.3(3.3)$ & $4.2(3.2)$ \\
HADS anxiety score (SD) & $5.6(3.6)$ & $4.5(3.7)$ \\
State anxiety score (SD) & $1.8(0.7)$ & $1.7(0.6)$ \\
\hline
\end{tabular}

$H A D=$ Hospital Anxiety and Depression Scale. intervention effect might be found. Using the NQuery sample size programme for $\alpha=0.05$ (type I error) and $\beta=0.2$ (type II error), a standardised effect size of 0.5 required 128 patients with complete results. Assuming a cluster size of up to 15 , and an intracluster correlation of 0.05 , the design effect was 1.55 and therefore 198 participants with complete data were required.

\section{RESULTS}

All 16 GPs were experienced (with more than 10 years in practice; eight had more than 20 years in practice), five were female, and one worked in a deprived inner city practice, but controlling for these features did not modify the estimates. Many patients could not be consented and entered into the study because of the limited time prior to their consultation. Most patients who could be approached with sufficient time agreed (Figure 1), with the remainder either not having the time or inclination to participate, and a very few not willing to participate because of the sensitive nature of the consultation. One hundred control patients and 124 intervention patients were recruited, of whom 190 (85\%; $n=92$ control, $n=98$ intervention) returned postconsultation questionnaires. Table 1 shows that the participant characteristics of the trial groups were reasonably balanced, and where slight differences occurred (for example, in the Hospital Anxiety and Depression Scale score) controlling for these made no difference to the estimates.

Table 2 shows that the intervention increased the overall satisfaction (MISS), mainly impacting distress-relief and relationship subscales, and improved patient perception of a communication and partnership approach, and health promotion. There were non-significant improvements in most other scales and a small non-significant reduction in enablement. No harms were reported in either group.

\section{DISCUSSION}

\section{Summary}

As far as the authors are aware, this study demonstrates for the first time that very brief communication training for GPs in a mixture of non-verbal elements such as avoiding non-verbal cut-offs and nonspecific verbal elements such as backchannelling, and personal reflection on videotapes of their consultations is likely to improve patients' perceptions of satisfaction, distress, a partnership approach, and health promotion. 


\section{Strengths and limitations}

A strength of this study, which investigated a simple intervention to improve communication, is that it could be rolled out relatively easily. The intervention is feasible and acceptable, does not require travel time for GPs, requires little time for generating videotapes, with minimal external facilitation, and pragmatically allows flexible viewing of videotapes (which was almost entirely left to GPs' discretion and interest). There was little attrition, and the results are unlikely to be explained by type I error for several reasons: the primary outcome was significant, there were several significant secondary findings, and nearly all the non-significant ones went in the same direction. The groups were generally well balanced and where they were unbalanced slightly, there was no evidence of confounding.

Weaknesses were also noted. The intervention was based on prior empirical data, and although brief, it was nevertheless a complex intervention (providing several suggestions and also reflection on videotapes) and so it is unclear what elements were the key to the success of the intervention. It is not clear which component of the intervention (KEPe Warm, viewing videotapes, or both) was the most powerful - to tease out which components are most important a large factorial trial would be the optimal design. Face-to-face input and interaction was minimal, so the training could have been made easier to encourage widespread implementation by using video presentations or by using the internet. ${ }^{13}$

The intervention has limitations for some participants who would have preferred a more individualised intervention, but this would have the major disadvantage of being much more time consuming and more difficult and costly to implement in practice. The attitudes towards changing were on the whole positive but the GPs, who were all established practitioners, reported that this was a difficult process. The negative results for some scales could be type II error, particularly as the sample did not quite reach the intended sample from the power calculations. There is likely to be some selection bias in this trial because GPs who took part were self-selected, and likely to be more interested in communication. The high baseline scores suggests that this may be the case, which may have limited the ability to demonstrate change due to ceiling effects, so the possible impact of the intervention may have been underestimated. The GPs were relatively experienced, so whether the intervention would be as useful among newly-qualified GPs is less clear. There was only one GP serving a deprived inner-city practice area, and although the groups were not balanced for GP characteristics, controlling for a range of GP features did not modify the estimates. GP participants who arrived with little time to consent may have had more time pressure and distress, so the sample may have included fewer people most likely to benefit from the intervention. The study has also not shown whether improving communication improves health status, although if distress is relieved it is likely to have some impact on a patient's quality of life and/or mental health, but a much larger study would be needed to assess health outcomes.

\section{Comparison with existing literature}

The quantitative results showed a moderate increase in perceived satisfaction, equivalent to shifting the distribution of satisfaction more than 10 centile points from the 50th centile to above the 60th centile, with a standardised mean difference of 0.32 . There was particularly improved relief of distress and improved perception of a partnership approach of the patients after consulting with GPs who had undertaken the intervention compared with those who had not. These results are in line with the prior systematic reviews, ${ }^{4,6,14}$ which found that promoting a patient-centred approach in consultations showed improvements in consultation processes and satisfaction la standardised effect size of 0.35 , which is very similar to the effect size reported here). ${ }^{6}$ However, most of the interventions reported in the systematic reviews were much more intensive, required significant input from external facilitators, did not address nonverbal skills, and were often not in typical primary care settings. ${ }^{4,6,14}$

\section{Implications for practice}

Engaging GPs in brief training of predominantly non-verbal and non-specific verbal elements of communication, and encouragement to reflect on their consultation videotapes should be considered. This is likely to improve patients' perception of satisfaction, distress, a partnership approach, and health promotion.

project team; Anna Jackson, Lino Wong, and Fiona Place lall medical students at the time).

\section{Discuss this article}

Contribute and read comments about this article: bjgp.org/letters 


\section{REFERENCES}

1. Beck RS, Daughtridge R, Sloane PD. Physician-patient communication in the primary care office: a systematic review. J Am Board Fam Pract 2002; 15: 25-38.

2. Little $P$, Everitt $H$, Williamson I, et al. Preferences of patients for patient centred approach to consultation in primary care: observational study. BMJ 2001; 322 468-472

3. Little $\mathrm{P}$, Everitt $\mathrm{H}$, Williamson I, et al. Observational study of effect of patient centredness and positive approach on outcomes of general practice consultations. BMJ 2001; 323: 908-911.

4. Griffin SJ, Kinmonth AL, Veltman MW, et al. Effect on health-related outcomes of interventions to alter the interaction between patients and practitioners: systematic review of trials. Ann Fam Med 2004; 2: 595-608.

5. Kinnersley P, Edwards A, Hood K, et al. Interventions before consultations to help patients address their information needs by encouraging question asking: systematic review. BMJ 2008; 337: a485.

6. Dwamena F, Holmes-Rovner M, Gaulden CM, et al. Interventions for providers to promote a patient-centred approach in clinical consultations. Cochrane Database Syst Rev 2012; 12: CD003267

7. Little $\mathrm{P}$, White $\mathrm{P}$, Kelly J, et al. Verbal and non-verbal behaviour and patient perception of communication in primary care: an observational study. Br J Gen Pract 2015; DOI:10.3399/bjgp15X685249.

8. Thomas KB. General practice consultations: is there any point in being positive? BrMed J 1987; 294: 1200-1202

9. Mead N, Bower P. Measuring patient-centredness: a comparison of three observation-based instruments. Patient Educ Couns 2000; 39: 71-80.

10. Hallam D, Doggett L, Wilkin AM. Measures of need and outcome for primary health care. Oxford: Oxford University Press, 1992.

11. Howie JG, Heaney DJ, Maxwell M, et al. Quality at general practice consultations: cross sectional survey. BMJ 1999; 319: 738-743.

12. Marteau TM, Bekker H. The development of a six-item short-form of the state scale of the Spielberger State-Trait Anxiety Inventory (STAI). Br J Clin Psychol 1992; 31(3): 301-306.

13. Little $\mathrm{P}$, Stuart B, Francis N, et al. Effects of internet-based training on antibiotic prescribing rates for acute respiratory-tract infections: a multinational, cluster, randomised, factorial, controlled trial. Lancet 2013; 382: 1175-1182.

14. Lewin SA, Skea ZC, Entwistle V, et al. Interventions for providers to promote a patient-centred approach in clinical consultations. Cochrane Database Syst Rev 2001; 4: CD003267. 